\title{
Registros de ataque y depredación sobre anfibios y reptiles de Limoncocha, Amazonía norte de Ecuador
}

\author{
Records of Attack and Predation on Amphibians and Reptils from \\ Limoncocha, Northern Amazon of Ecuador
}

Salomón M. Ramírez-Jaramillo1*; Jesenia M. Castillo-Merino²

1 Investigador Independiente. Santa Isabel, Quito, Ecuador.

* Correo electrónico: kp-7sz@hotmail.com

2 Ministerio del Ambiente. Guardaparque de la Reserva Biológica Limoncocha. Correo electrónico: jeseniacastillo2@gmail.com

\section{RESUMEN}

Con el propósito de generar información sobre la historia natural y el comportamiento de la fauna terrestre en la localidad de Limoncocha, se realizaron recorridos de muestreo diurno y nocturno durante agosto y octubre de 2019. Mediante registros fotográficos damos a conocer cuatro eventos depredatorios de la Amazonia norte de Ecuador. Se presentan los registros de Leptophis cupreus suspendida de un árbol ingiriendo a Boana appendiculata; Cyanocorax violaceus depredando a Dendropsophus reticulatus; dos ocasiones donde Ctenus sp. capturó juveniles de Allobates insperatus; $y$ una ocasión en que Milvago chimachima llevaba en sus garras a Epicrates cenchria. Estos registros de interacciones entre especies son inéditos para la Amazonia norte de Ecuador.

Palabras clave - Comportamiento de depredación, historia natural, interacciones tróficas.

\begin{abstract}
With the purpose of generating information about the natural history and behavior of the terrestrial fauna in the locality of Limoncocha, day and night sampling rounds were carried out during August and October 2018. Through photographic records we

Ref. bibliográfica: Ramírez-Jaramillo, S. M.; Castillo-Merino, J. M. "Registros de ataque y depredación sobre anfibios y reptiles de Limoncocha, Amazonía norte de Ecuador". Acta zoológica lilloana 64 (1): $65-72$. Fundación Miguel Lillo, Tucumán, Argentina. D.O.I.: https://doi.org/10.30550/j.azl/2020.64.1/6

> Recibido: 3 de junio de 2019 - Aceptado: 2 de abril de 2020

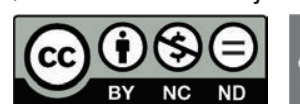


reveal four predatory events in the northern Amazon of Ecuador. They correspond to Leptophis cupreus hanging from a tree ingesting Boana appendiculata; Cyanocorax violaceus preying on Dendropsophus reticulatus; two occasions where Ctenus sp. caught juveniles of Allobates insperatus; and an occasion when Milvago chimachima carried Epicrates cenchria in its claws. This record of interactions between species is the first of its kind for the northern Amazon of Ecuador.

Keywords - Predation behavior, natural history, trophic interactions.

La Amazonía ecuatoriana comprende un gran acervo de biodiversidad dentro de un bioma complejo y cambiante, en donde las especies de flora y fauna que habitan en esta región interactúan constantemente (García, Parra, Mena, 2014). La herpetofauna amazónica está conformada por aproximadamente 358 especies de anfibios (184) y reptiles (174) (Ron, Merino Viteri, Ortiz, 2019; Torres Carvajal, Pazmiño Otamendi, Salazar Valenzuela, 2020). Sin embargo, se conoce poco acerca de las interacciones relacionadas con la herpetofauna amazónica. Por tal motivo, mediante registros fotográficos se muestra información novedosa acerca de cuatro sucesos de ataque y depredación ocurridos sobre la herpetofauna en la localidad de Limoncocha.

Las observaciones se realizaron en la localidad de Limoncocha $\left(00^{\circ} 24^{\prime} \mathrm{S}, 76^{\circ} 37^{\prime}\right.$ W), cantón Shushufindi, provincia de Sucumbíos; dentro del ecosistema de Bosque siempreverde de tierras bajas del Aguarico-Putumayo-Caquetá (Ministerio del Ambiente del Ecuador [MAE] (2013)). Durante los recorridos realizados en agosto y octubre de 2018, se observaron y documentaron mediante fotografías cuatro eventos de depredación.

El 11 de octubre de 2018, a las 14:00 horas, se registró la depredación de Leptophis cupreus Cope, 1868, sobre Boana appendiculata Caminer y Ron, 2020. A 1,70 m de altura, se hallaba Leptophis cupreus envuelta entre las ramas de la vegetación y verticalmente suspendida aproximadamente un tercio de su cuerpo, sostenía con sus mandíbulas a Boana appendiculata, que realizaba movimientos, mientras la engullía lentamente (Figura 1A-B). Se observó durante 25 minutos aproximadamente como ingería su presa. Se conoce poco sobre la dieta de este ofidio, registros previos reportan ranas (Pristimantis) entre sus presas (Albuquerque y McDiarmid, 2010).

El 20 de agosto de 2018, entre las 12:35 y 12:36 horas, se observó a Cyanocorax violaceus Du Bus de Gisignies, 1847, aproximadamente a $20 \mathrm{~m}$ de alto, posado sobre un árbol de Cecropia sciadophylla Mart. 1841, alimentándose de un individuo de Dendropsophus reticulatus Jiménez de la Espada, 1870. Cyanocorax violaceus sostenía con su garra derecha a Dendropsophus reticulatus, y lo desmembraba con su pico, luego lo cambió de garra, mientras continuaba desmembrándolo hasta consumirlo totalmente (Figura 2). Sobre la dieta en esta especie de córvido se conoce que son omnívoros y oportunistas, se alimentan principalmente de frutos, insectos y huevos, incluyendo lagartijas (Hilty, 2003; Lopes, Fernandes, Marini, 2005). El presente registro constituye el primer reporte de depredación de Cyanocorax violaceus sobre una especie de anuro. 

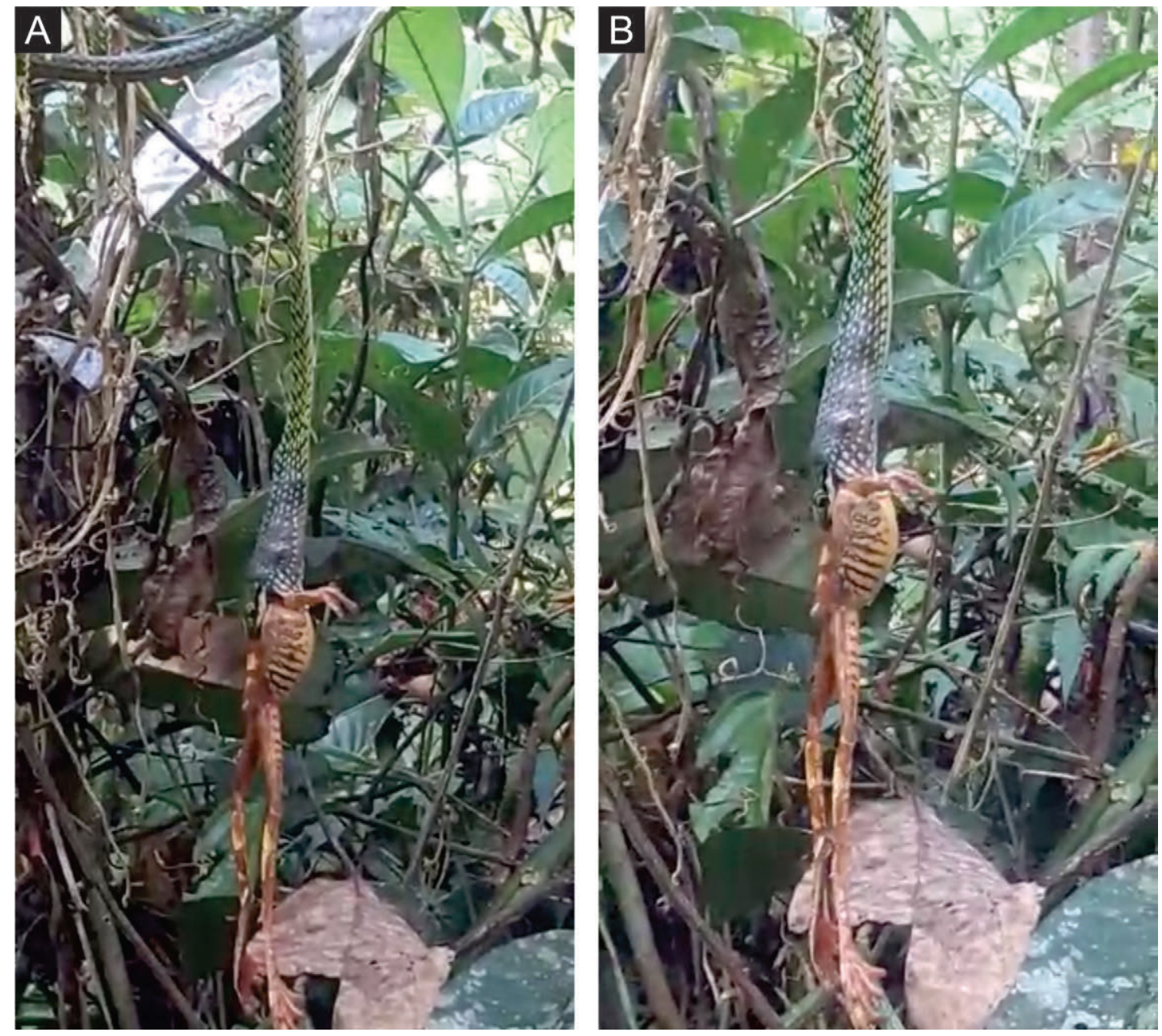

Figura 1. Depredación de Leptophis cupreus sobre Boana appendiculata.

Figure 1. Predation of Leptophis cupreus on Boana appendiculata.

El 20 de agosto de 2018, entre las 9:00 y 11:30 horas, se registraron 147 individuos juveniles de Allobates insperatus Morales 2002, mientras que en la noche entre las 19:30 y 22:30 horas se registraron nueve individuos juveniles. No se registraron individuos adultos. A las 19:53 horas se observó el brinco de una araña lobo Ctenus sp. Walckenaer, 1805, para capturar a una rana juvenil de Allobates insperatus (Figura 3A), posteriormente acomodó a su presa hasta sostenerla por su parte anterior dorsal, hasta las 20:04 horas (Figura 3B). Posteriormente a las 22:26 horas se observó a otro individuo de Ctenus sp., que había atrapado un juvenil de Allobates insperatus (Figura 3C), luego lo manipuló hasta sostenerlo por su parte anterior ventral, hasta las 22:30 horas (Figura 1D). En ambos casos, las observaciones no detectaron la consumación de su presa. Posteriormente las arañas se perdieron sosteniendo a su presa y dando brincos entre la hojarasca. Se conoce que las arañas del género Ctenus cazan mayormente de noche, y capturan presas mediante pequeños saltos y "mordeduras". Registros alimenticios previos indican que las arañas del género Ctenus consumen tetrápodos (Höfer, Brescovit, Gasnier, 1994; Melo Sampaio, Bezerra de Lima, Lima, 

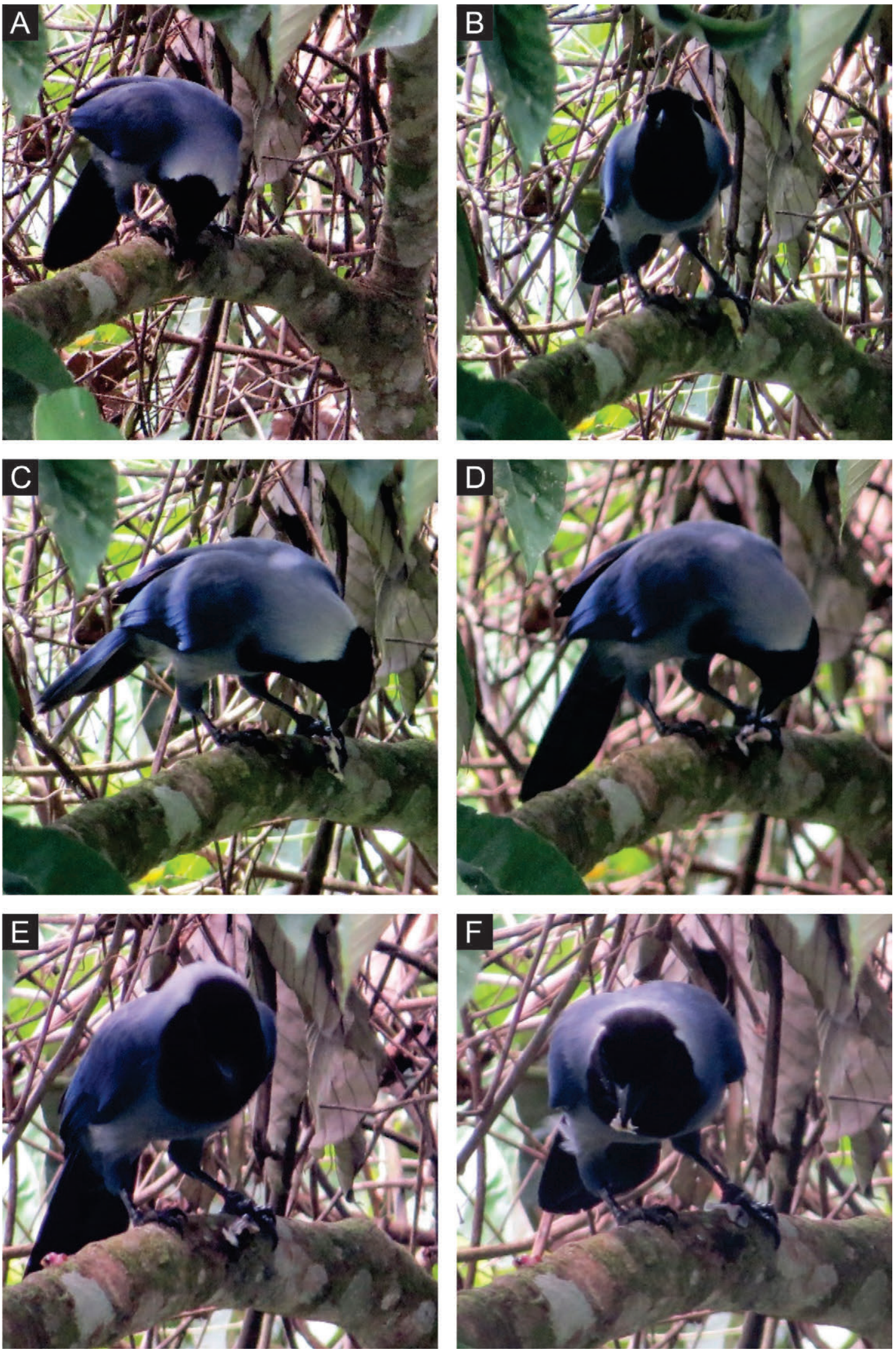

Figura 2. Depredación de Cyanocorax violaceus sobre Dendropsophus reticulatus.

Figure 2. Predation of Cyanocorax violaceus on Dendropsophus reticulatus. 

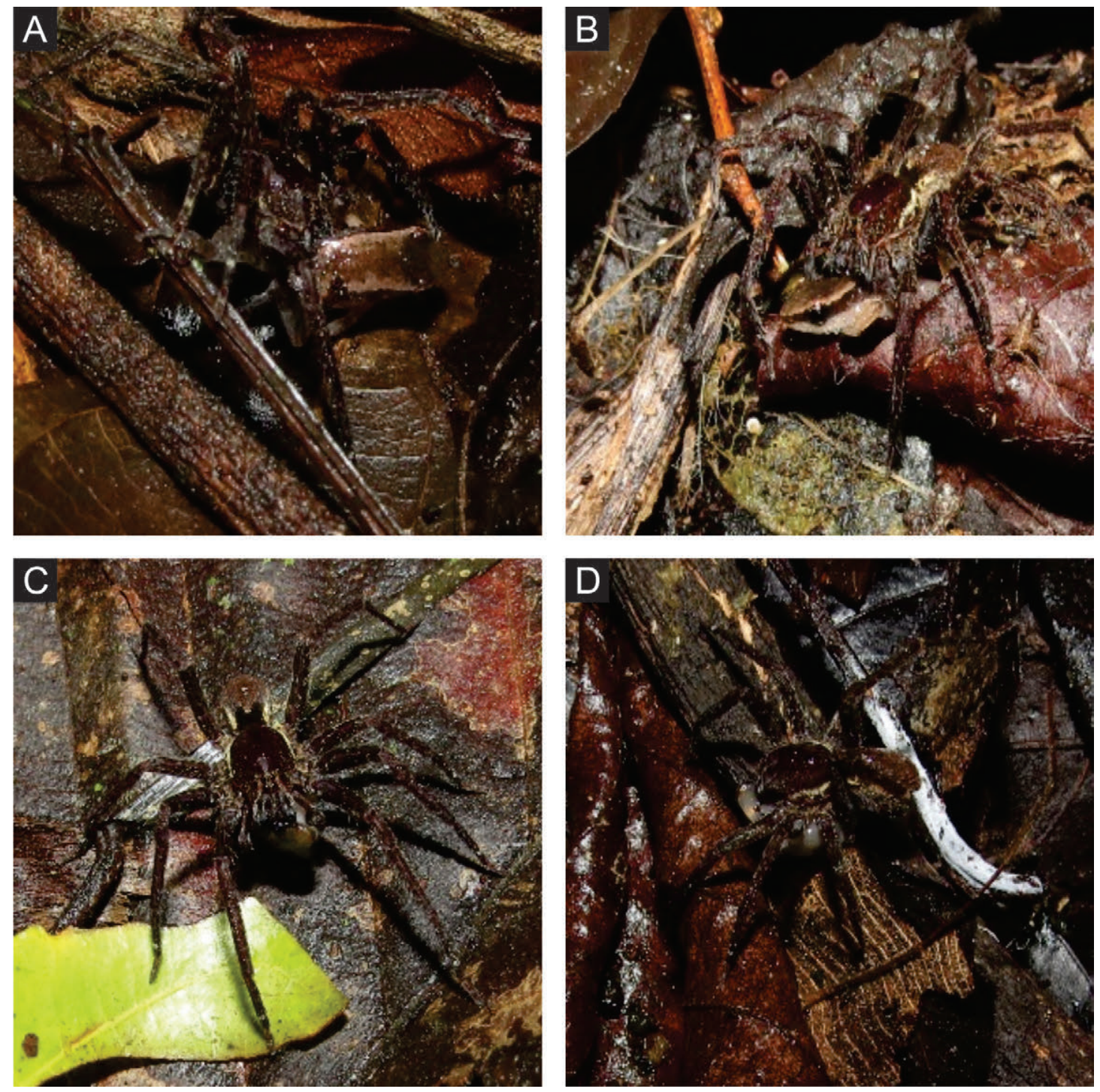

Figura 3. Dos sucesos de Ctenus sp., depredando sobre Allobates insperatus.

Figure 3. Two events of Ctenus sp. preying on Allobates insperatus.

Monteiro, Da Silva, 2017; Willemart y Lacava, 2017). Este registro constituye el primer reporte para Ecuador, de una araña Ctenus depredando a un anuro.

El 27 de agosto del 2018, a las 8:56 horas se avistó un adulto de Milvago chimachima Vieillot, 1816, llevar en sus garras a un juvenil de Epicrates cenchria Linnaeus, 1758, y posarse sobre un árbol de Cecropia sciadophylla Mart., a 5 metros de altura. Inmediatamente percató la presencia del observador, soltó a su presa abandonándola en una rama del árbol, y se posó en una rama opuesta. Inclinándose hacia adelante, miró al observador (Figura 4A), luego dio pequeños pasos de un lado a otro (Figura 4B). La presa abandonada estaba parcialmente consumida por la rapaz ya que tenía amputado un extremo (Figura 4C-D). A las 11:05 horas la presa abandonada seguía en su sitio. Sin embargo, al día siguiente, a las 9:00 horas, no se detectó a la presa abandonada. 

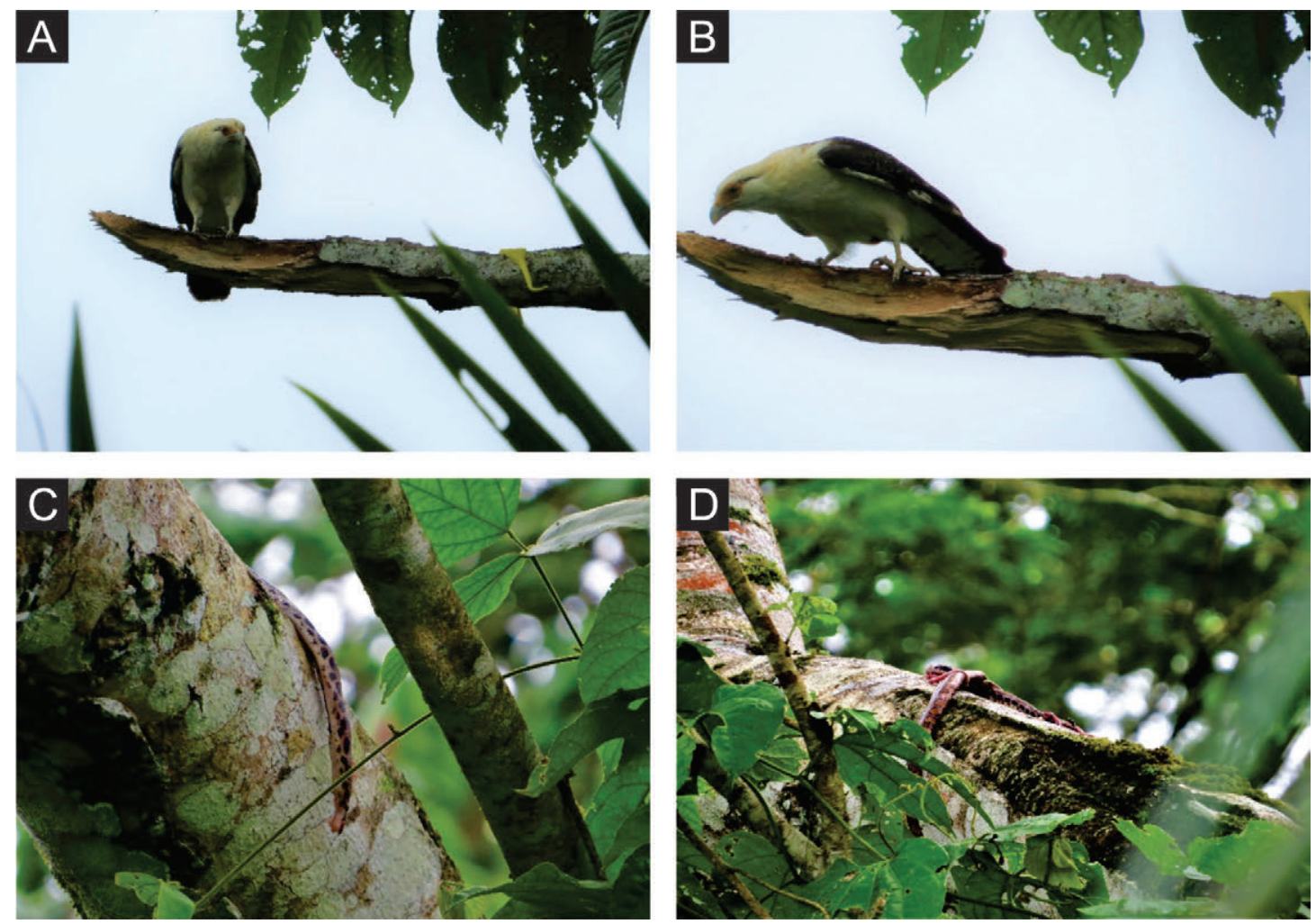

Figura 4. Depredación de Milvago chimachima sobre Epicrates cenchria.

Figure 4. Predation of Milvago chimachima on Epicrates cenchria.

Milvago chimachima presenta hábitos de vuelo bajo y para buscar presas suele frecuentar áreas abiertas o semiabiertas cercanas a los ríos, campos de fútbol, pastizales y ganado. Suele posarse en pequeños montículos de tierra o maderas, y en la copa de los árboles. También suele forrajear en el suelo, caminar y dar pequeños saltos para atrapar presas vivas más pequeñas. Su dieta es omnívora (artrópodos, peces, anfibios, aves y mamíferos), es carroñera e incluso garrapatera, y también suele consumir frutos y semillas (Ferguson Lees y Christie, 2001; Hilty, 2003; Ridgely y Greenfield, 2006).

\section{AGRADECIMIENTOS}

Al Ministerio del Ambiente por otorgarnos los permisos de investigación $\mathrm{N}^{\circ}$ MAEDNB-CM-2017-0062 y 0014-18IC-FAU-DNB/MA. A Esteban Calvache por su ayuda en la confirmación del género del arácnido. A Mauricio Ortega por su dirección y gestión para el levantamiento de información de campo durante el proyecto. A nuestras familias por su constante apoyo. 


\section{FINANCIAMIENTO}

Las salidas de campo fueron financiadas por la Corporación Alemana dentro del proyecto "Sistema Nacional de Monitoreo de la Biodiversidad - SINMBIO", y el Ministerio del Medio Ambiente.

\section{PARTICIPACIÓN}

Los autores realizaron las observaciones de campo, tomaron fotografías, identificaron los individuos y redactaron el manuscrito.

\section{CONFLICTOS DE INTERÉS}

Los autores declaramos explícitamente que no existen conflictos de interés entre nosotros o con terceros.

\section{LITERATURA CITADA}

Albuquerque, N. R., McDiarmid, R. W. (2010). Redescription of Leptophis cupreus (Cope) (Serpentes, Colubridae), a rare South American colubrine snake. Papéis Avulsos de Zoologia, 50, 375-384.

Ferguson Lees, J., Christie, D. F. (2001). Raptors of the World. New York: Houghton Mifflin Company.

García, M., Parra, D., Mena, P. (2014). El país de la biodiversidad: Ecuador. Quito: Fundación Botánica de los Andes, Ministerio del Ambiente y Fundación EcoFondo.

Hilty, S. L. (2003). Birds of Venezuela (2 ed.). Princeton University Press.

Höfer, H., Brescovit, A. D., Gasnier, T. R. (1994). The wandering spiders of the genus Ctenus (Ctenidae, Araneae) of Reserva Ducke, a rainforest reserve in central Amazonia. Andrias, 13, 81-98.

Lopes, L. E., Fernandes, A. M., Marini, M. Â. (2005). Predation on vertebrates by Neotropical passerine birds. Lundiana, 6, 57-66.

Melo Sampaio, P. R., Bezerra de Lima, R., Lima, J. M., Monteiro, C., Da Silva, R. (2017). Adenomera hylaedactyla, predation. Herpetological Review, 48, 602.

Ministerio del Ambiente del Ecuador (MAE). (2013). Sistema de Clasificación de los Ecosistemas del Ecuador Continental. Quito, Ecuador: Subsecretaría de Patrimonio Natural.

Ridgely, R. S., Greenfield, P. J. (2006). Aves del Ecuador. Guía de Campo. Quito: Fundación de Conservación Jocotoco.

Ron, S. R., Merino Viteri, A., Ortiz, D. A. (2019). Anfibios del Ecuador. Versión 2019.0. Museo de Zoología, Pontificia Universidad Católica del Ecuador: Disponible: https://bioweb.bio/faunaweb/amphibiaweb. Acceso el 27 de marzo de 2020. 
Torres Carvajal, O., Pazmiño Otamendi, G., Salazar Valenzuela, D. (2020). Reptiles del Ecuador. Versión 2020.0. Museo de Zoología, Pontificia Universidad Católica del Ecuador. Disponible: https://bioweb.bio/faunaweb/reptiliaweb. Acceso el 27 de marzo de 2020.

Willemart, R. H., Lacava, M. (2017). Foraging strategies of cursorial and ambush spiders. In: Behaviour and Ecology of Spiders: Contributions from the Neotropical Region (227-245). Viera, Gonzaga (Edits.). Cham, Switzerland: Springer. doi: https://doi.org/10.1007/978-3-319-65717-2. 